Adaptive Energy-based Bilinear Control of First-Order 1-D Hyperbolic PDEs: Application to a One-Loop Parabolic Solar Collector Trough

Sarah Mechhoud, Taous-Meriem Laleg-Kirati

PII: S0016-0032(17)30634-8

DOI: 10.1016/j.jfranklin.2017.12.003

Reference: $\quad$ FI 3254

To appear in: $\quad$ Journal of the Franklin Institute

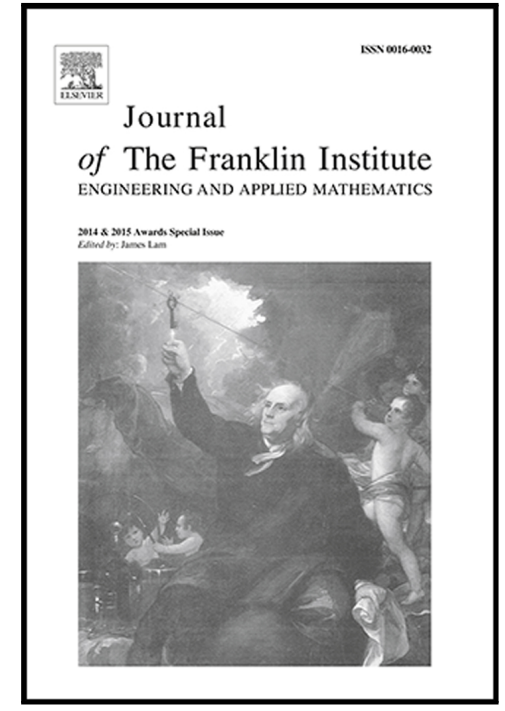

Received date: $\quad 14$ March 2017

Revised date: $\quad 5$ October 2017

Accepted date: $\quad 6$ December 2017

Please cite this article as: Sarah Mechhoud, Taous-Meriem Laleg-Kirati, Adaptive Energy-based Bilinear Control of First-Order 1-D Hyperbolic PDEs: Application to a One-Loop Parabolic Solar Collector Trough, Journal of the Franklin Institute (2017), doi: 10.1016/j.jfranklin.2017.12.003

This is a PDF file of an unedited manuscript that has been accepted for publication. As a service to our customers we are providing this early version of the manuscript. The manuscript will undergo copyediting, typesetting, and review of the resulting proof before it is published in its final form. Please note that during the production process errors may be discovered which could affect the content, and all legal disclaimers that apply to the journal pertain. 


\title{
Adaptive Energy-based Bilinear Control of First-Order 1-D Hyperbolic PDEs: Application to a One-Loop Parabolic Solar Collector Trough
}

\author{
Sarah Mechhoud*, Taous-Meriem Laleg-Kirati \\ Computer, Electrical and Mathematical Sciences and Engineering Division, King Abdullah University \\ of Science and Technology, \\ Thuwal 23955-6900, Saudi Arabia
}

\begin{abstract}
\end{abstract}
In this paper, the adaptive bilinear control of a first-order 1-D hyperbolic partial differential equation (PDE) with an unknown time-varying source term is investigated where only boundary measurements are available. By means of boundary injection, the bilinear adaptive law is developed in the Lyapunov approach. It consists of a state observer and an input adaptation law combined with a bilinear control method derived using an energy-like principle. Both global asymptotic practical convergence of the tracking error and input-to-state stability of the system are guaranteed. A potential application of this control strategy is the one-loop solar collector parabolic trough where the solar irradiance is the unknown input (source term) and the flow rate is the control variable. The objective is to drive the boundary temperature at the outlet to track a desired profile. Simulation results are provided to illustrate the performance of the proposed method. Keywords: Distributed parameter systems; bilinear control; state and input estimation; adaptive control; output feedback control; practical asymptotic stability.

\section{Introduction}

Transport processes characterize many industrial, physical and chemical systems ranging from heat transport mechanisms to the transport of species in biology sys-

\footnotetext{
* Corresponding author

Email addresses: sarra.mechhoud@kaust.edu.sa (Sarah Mechhoud), taousmeriem.laleg@kaust.edu.sa (Taous-Meriem Laleg-Kirati)

Preprint submitted to The Journal of the Franklin Institute
} 
tems. Transport systems are distributed parameter systems (DPS) which are generally described by hyperbolic PDEs and especially by first-order PDEs when the advection phenomenon dominates the diffusion and the reaction ones. Advection expresses the transport or movement of a property like heat or of a quantity like a material by the bulk motion of a fluid. The advection equation is a first-order hyperbolic PDE and is often used to explain systems of conservation laws or systems of balance laws. For example, to name a few, heat transport in plug-flow reactors, traffic transport and gas transport in pipelines are some real-world applications described using advection equations. In this paper we consider the heat transport in solar collector parabolic troughs.

Renewable energy resources like the solar energy are regarded as promising alternatives to fossil fuels. A parabolic solar collector is a DPS/engineering system designed to collect and store the sunlight energy as a part of a solar power plant. Using energy balance laws, heat transport in a one-loop solar collector field is described by a bilinear advection equation. When the heat losses are neglected, the time-varying source term (input) represents the solar irradiance. The fluid flow rate is the control variable by which the outlet temperature of the loop is maintained at a prescribed level in spite of the surrounding disturbances [1].

Solar thermal power generation is a highly appealing source of electricity. However, there are some issues facing the control of this energy resource. One of them is the solar irradiance. Due to its intermittence (variation with respect to the weather conditions, the temporal dust concentration, the vapour concentration, etc.) it is difficult to predict its behavior and this affects the normal and smooth operation of the solar collector plant. Some mechanical or electrical sensors are available locally and at some period of time to measure the sun iryadiance. However, providing each collector with a sun tracking sensor is a capital intensive enterprise. This will add to the already high cost of solar energy conversion systems.

For economical (solar energy cost) and technical (control of the solar collector and its tracking system) purposes, it is important to estimate the solar irradiance to overcome these difficulties and build an efficient solar plant. In the literature, some empirical and numerical models and estimators of the solar irradiance already exist, however they are based on other solar irradiance measurements (atmospheric conditions, meteorological 
data, etc., see[1, Chap. 2] for more details).

Many control methods have been proposed for the control of the solar collector loop, a survey may be found in [1]. However most of this methods were addressed in the early lumping approach (discretizing first and controlling later) which is not relevant for hyperbolic PDEs, since there is no distinct gap between fast and slow modes [2, Chap. 2, p.11]. Therefore, a discrete approximate model may differ significantly from the original PDE [3]. In this paper, a new adaptive control strategy is proposed to control the solar collector model in the late lumping approach, i.e by considering the control problem in the infinite dimensional framework. Recent years have seen extensive studies on observer design and control techniques of first-order hyperbolic systems in the infinite dimensional framework (see [4], [5], [6] and references therein). One of the pioneer work in this field was Christofides et al.'s paper in [7]. It addressed the problem of feedback control of linear and quasi-linear hyperbolic PDEs using geometric control and distributed inputs and measurements. Then, in [8], robust control of hyperbolic PDEs was dealt with while assuming available point-wise actuators and measurements. Boundary control of hyperbolic PDEs was considered using either Lyapunov design ([9] and references therein) or the powerful backstepping method ([5], [6] and references therein). This latter, backstepping method, was then extended to the control problem of hyperbolic PDEs with unknown constant and functional parameters ([10], [11] and references therein). Fuzzy control design was also proposed for the control of nonlinear hyperbolic PDEs using distributed inputs (see references [12], [13] for more details). However, to our best knowledge, few research work have been dedicated to the control problem of PDEs with unknown time-varying parameters (e.g: [14]). Moreover, the aforemenfioned methods dealt with distributed, boundary or in-domain control inputs. The problem of bilinear control of first-order PDEs has attracted less attention and was generally investigated using either geometric or optimal control. The geometric control is constrained by its inability to check analytically the stability of the zero dynamics [2]. Whereas, the optimal control problem in bilinear systems is complex and leads in practice to suboptimal control laws. In [15], a new observer-based bilinear control approach was proposed for the solar collector model. It was able to guarantee the exponential convergence of the tracking error using direct measurements of the solar irradiance. 
In this paper, a new adaptive controller based on the energy-like control principle proposed in [16] for the heat transport model of the solar collector combined with the adaptive estimation method of time-varying parameters proposed in [14] is considered. The solar irradiance is assumed to be unknown and only the boundary measurements of the temperature at the outlet of the tube are taken into consideration. Unlike most robust control studies ([17] and references therein) in this method, we do not assume that the bounds on the unknown input or its time-variation is known.

The contributions of this work are mainly (i) the design of a new adaptive controlmethod for first-order 1D hyperbolic PDEs, (ii) where the proposed adaptive controller does not require a reference model as it is usually the case in MRAC methods and guarantees the stability of the system and the global asymptotic practical reference tracking. (iii) The estimation of the time-varying source term (solar irradiance) which is not only necessary for the control objective but also useful in the selection of the target state. Note that the method developed herein may find application in various systems that share the same description like spray driers, high way traffic, heat exchangers, etc.

This paper is organized as follows. In Section 2, the solar collector model and the conditions of its well-posedness are formulated. The reachability conditions are given in Section 3. In Section 4, the adaptive control and the proof of its global asymptotic practical convergence are presented. Simulation results are depicted in Section 5.

\section{Notations and definitions}

Throughout this paper, we denote by $L_{2}$ the usual space of square integrable function endowed with its standard norm $\|\cdot\|_{2} . H^{s}(\Omega)$ is the Hilbert space defined by

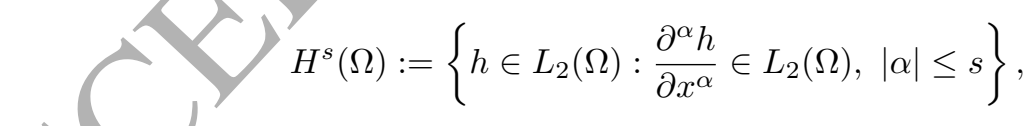

where $\alpha$ is the multi-index. $L_{\infty}$ is the space of bounded functions equipped with its usual norm 1$)\left.\right|_{\infty}$.

Definition of a $C_{0}-$ semigroup

In the following, we present the general definition of a $C_{0}$-semigroup generated by a time-invariant operator $A$. 
Definition: A $C_{0}$-semigroup (or a strongly continuous semigroup) of a one-parameter family $U: \mathbb{R}^{+} \longrightarrow L(X)$ satisfyies

- $U(0)=I($ the identity on $X)$,

- $U(t+s)=U(t) U(s)$

- $\lim _{t \rightarrow 0^{+}} U(t) f=f$ for each $f \in L(X)$ with respect to the norm on $X$.

where $L(X)$ is the space of bounded linear operators on the Banach space $X$. If in addition, $\|U\|<1$ then $T$ is said to be a $C_{0}$-semigroup of contractions.

A $C_{0}$-semigroup can be seen as a generalization of the exponential function to solve differential equations in Banach spaces. In the case of a time-varying operator $A(t)$, the generated $C_{0}$-semigroup is of two parameters, i.e. $U(t, s), 0 \leq s \leq t$. More details on $C_{0}$-semigroups can be found in Pazy's book [18], Chap. 1 and 5 .

We recall here the general definitions of reachability and controllability.

Definition: Let us consider a system described by the following state-space representation

$$
\dot{X}(t)=\varphi\left(X(t), u(t) ; X_{0}\right), \quad X(0)=X_{0},
$$

where $X$ is the state and $u$ is the control input.

The set $W^{+}\left(X_{1}\right)$ of reachable states $X_{1}$ at time $t_{1}$ starting from $X_{0}$ is defined by

$$
W^{千}\left(X_{1}\right)=\left\{X_{1}: X_{1}=\varphi\left(X\left(t_{1}\right), u(.) ; X_{0}\right), u \in \mathcal{U}\right\} .
$$

where $\mathcal{U}$ is the set of admissible inputs.

The set $W f\left(X_{0}\right)$ of eontrollable states to $X_{1}$ at time $t_{1}$ is given by

$$
W^{-}\left(X_{0}\right)=\left\{X_{0}: X_{1}=\varphi\left(X\left(t_{1}\right), u(.) ; X_{0}\right), u \in \mathcal{U}\right\} .
$$

More details and discussion on the difference between these two notions can be found in [19], section 2.5 .

\section{Problem formulation}

Using energy balance laws, the solar collector field can be described by the heat transport of the circulating fluid. Moreover, if the heat losses are neglected, the following 
model is obtained (for more details see [1] and references therein)

$$
\left\{\begin{array}{l}
\left.\left.\left.\left.\frac{\partial T}{\partial t}(x, t)=-q(t) \frac{\partial T}{\partial x}(x, t)+f(t), \quad x \in \Omega=\right] 0,1\right], \quad t \in\right] 0, t_{f}\right] \\
T(x=0, t)=g(t), \quad t \in\left[0, t_{f}\right] \\
\left.\left.T(x, t=0)=T_{0}(x), \quad x \in\right] 0,1\right] .
\end{array}\right.
$$

with

$$
f(t)=\frac{\eta_{0} G}{\rho c S} I(t)
$$

and where $T(x, t)$ is the fluid temperature, $q(t)$ is the fluid pump volumetric flow rate, $\eta_{0}$ is the mirror optical efficiency, $\rho$ is the fluid density, $c$ is the specific heat capacity, $S$ is the cross-sectional area and $I(t)$ is the solar irradiance. The normalized space and time variables are denoted $x$ and $t$ respectively, where $t_{f}$ is the normalized final time. The second and third equations in system (1) define, respectively, the boundary and initial conditions guaranteeing the zero-order compatibility condition

$$
g(t=0)=T_{0}(x=0) \text {. }
$$

The output equation is given by

$$
y(t)=T(1, t),
$$

which states that the only available temperature measurements (sensor) are located at the tube outlet where $x=1$.

\subsection{Well-posedness of the solar model}

As demonstrated in [20], the following proposition gives conditions on the existence, uniqueness and regularity of the solution of equation (1).

Proposition 2.1. For all inputs $q$ strictly positive in $H^{s}\left(0, t_{f}\right), f \in L^{\infty}\left(0, t_{f}\right), g \in$ $H^{s}\left(0, t_{f}\right)$ and $T_{0} \in H^{s}(0,1)$, equation (1) has a unique solution in $C^{r}\left(0, t_{f} ; H^{s-r}(\Omega)\right)$ provided that $r$-compatibility conditions are satisfied. 
Proof. The proof of this proposition is directly obtained by applying Theorem 3.1 of [20]. Besides, in system (1), the dynamic operator $A(t)$ is defined by

$$
A(t)=-q(t) \frac{\partial}{\partial x}
$$

Its domain is independent of time and is given by

$$
D(A(t))=H_{0}^{1}(0,1):=\left\{h \in L_{2}(0,1): \frac{\partial h}{\partial x} \in L_{2}(0,1), h(0)=0\right\}
$$

The flow rate $q(t)$ is positive by definition, this states that the direction of the flow is from the left (tube's inlet) to the right (tube's outlet). Thus the operator $A(t)$ is an infinitesimal generator of a $C_{0}$-semigroup of contraction and the family $\{A(t)\}_{t \in\left[0, t_{f}\right]}$ is exponentially stable [21] and [18], Chap. 5. Therefore the requirement stated in Theorem 3.1 by [20] is fulfilled.

Remark 2.1 The $r$-compatibility condition imposes conditions on the smoothness of the data $\left(f, g, T_{0}\right)$ in order to ensure that the solution of equation $(1)$ is smooth enough. In other words, r-compatibility condition ensures higher order continuity of derivatives of the PDE solution. Especially it guarantees $C^{r}$ regularity of the PDE solution. Thus, the zero-order compatibility condition is a particular case which implies the continuity $\left(C^{0}\right)$ of the PDE solution (a technical definition of the $r$-compatibility condition can be found in $[20])$.

\subsection{Statement of the control problem}

Let $T^{*}(1, t)$ be the target temperature profile at the solar collector outlet. The objective of this paper is to synthesize a control law $q(t)$ such as $q_{\min }<q(t)<q_{\max }$, that drives the outlet temperature $T(1, t)$ towards the target state $T^{*}(1, t)$ in a suitable norm (which will be specified later) while assuming that the inlet temperature is known and the only available measurements are those of the outlet temperature at $T(x=1, t)$.

To satisfy the control objective, we use the following variable $\delta(t)$ which is the average tracking error $[16]$

$$
\delta(t)=\int_{0}^{1}\left(T^{*}(x, t)-T(x, t)\right) d x, \quad t \geq 0,
$$

Remark 2.2 Since $\delta(t)$ is the average tracking error, driving $\delta(t)$ towards zero will not lead directly to the convergence of the outlet temperature tracking error. Some 
conditions have to be considered in order to satisfy this convergence. The following proposition states these conditions.

Proposition 2.2. The average tracking error $\delta(t)$ given in (6) represents the $L_{1}-$ norm of the tracking error if

$$
\left.\left.T^{*}(x, t=0) \geq \sup _{x \in \Omega}\{T(x, t=0)\}, \forall x \in\right] 0,1\right],
$$

where $T^{*}(x, t=0)$ is the initial condition of the target system generating the desired temperature profile $T^{*}(x, t)$ and $T(x, t=0)$ is the initial condition of system (1).

Proof. The target system is defined by PDE (1) by choosing the initial condition to be $T^{*}(x, t=0)$ while the volumetric flow rate is denoted by $q^{*}(t)$ and the boundary condition is assumed to be equal to PDE's (1) boundary condition. Using the method of characteristics, the analytic solution of PDE (1) is given by

$$
T(x, t)=\left\{\begin{array}{l}
T_{0}\left(x-\int_{0}^{t} q(\tau) d \tau\right)+\int_{0}^{t} f(\tau) d \tau, \quad x \geq \int_{0}^{t} q(\tau) d \tau \\
g(t-\gamma)+\int_{0}^{\gamma} f(\tau) d \tau, \quad x<\int_{0}^{t} q(\tau) d \tau
\end{array}\right.
$$

where

$$
\gamma=\int_{0}^{x} \frac{d \alpha}{q(r(\alpha))}
$$

and $r(x)$ is the inverse of the characteristic curve equation $x(t)$, for more details on the method of characteristics see [22], Chap. 2. Using the analytic solution in (8), the analytic expression of $\delta(t)$ is given by

$$
\delta(t)=\int_{0}^{1}\left(T_{0}^{*}\left(x-\int_{0}^{t} q^{*}(\tau) d \tau\right)-T_{0}\left(x-\int_{0}^{t} q(\tau) d \tau\right)\right) d x
$$

and thus if the condition given in equation (7) is satisfied the integrand in the definition of $\delta(t)$ is positive and thus the tracking error $\left(T^{*}(x, t)-T(x, t)\right)$ is positive and $\delta(t)$

represents the $L_{1}$-norm of the tracking error.

Remark 2.3 The convergence towards zero and the positivity of the integrand of $\delta(t)$, i.e. $\left(T^{*}(x, t)-T(x, t)\right)$ guarantee the convergence of the distributed tracking error $\left(T^{*}(x, t)-T(x, t)\right)$ and consequently the convergence of the outlet tracking error 


$$
\begin{aligned}
& \left(T^{*}(x=1, t)-T(x=1, t)\right) \text {, i.e } \\
& \qquad \delta(t) \underset{t \rightarrow \infty}{\longrightarrow} 0 \Longleftrightarrow\left(T^{*}(x=1, t)-T(x=1, t)\right) \underset{t \rightarrow \infty}{\longrightarrow} 0 .
\end{aligned}
$$

Since the plant's initial condition $T_{0}(x)$ is known, throughout this work, we assume that the target profile $T^{*}$ is selected such as condition (7) is satisfied.

\section{Reachability of the solar collector model}

In the solar collector model, the flow rate $q(t)$ is the control variable and thus system (1) is a bilinear first-order hyperbolic PDE. The main result on the controllability of such systems is due to the pioneering work [23] where Ball et al. showed that exact controllability of bilinear PDEs cannot be achieved and only approximate controllability can be obtained, i.e. we can never expect to control system (1) to an open neighborhood of the initial state $T_{0}(x)$. Therefore, in this work we consider the reachability problem rather than the controllability one. Rather then looking for the set of states $T_{0}(x)$ from which we can reach a target state $T^{*}$ using an input $q$ (controllability), we seek the set of states $T^{*}$ that we can reach starting from $T_{\varnothing}(x)$ using input an $q$ (reachability).

In order to take into account the physical characteristics of the flow rate $q(t)$ and the solar irradiance $f(t)$ the following assumptions are adopted.

\section{Assumptions:}

(i) $q \in Q_{q}=\left\{q \in C^{0}(0, t): 0<q_{\min } \leq q(t) \leq q_{\max }\right\}$.

(ii) $f \in Q_{f}=\left\{f \in L^{2}(0, t): 0<f(t)<\infty\right.$ and $\left.|\dot{f}|<\infty, \forall t \geq 0,\right\}$.

(iii) $T_{0} \in L^{2}(0,1)$.

(iv) $g \in L^{2}(0, t)$.

These assumptions describe the fact that the flow rate $q(t)$ is lower bounded to ensure good operation conditions of the solar plant and upper bounded due to the physical limitations of the fluid pump [1]. The source term $f(t)$ is positive and bounded since the solar irradiance is positive by definition and the heat losses are neglected. Assumptions 
(iii) and (iv) stress out that the initial and boundary conditions are of finite energy.

We can now claim our reachability result in the following proposition.

Proposition 3.1. Given $T^{*}$ a target state in $C^{0}\left(0, t_{f} ; L^{2}(0,1)\right)$, if assumptions (ii)-(iv) are satisfied then there exists a control $q^{*} \in C^{0}\left(0, t_{f}\right)$ such as the solution of system (1) satisfies

$$
T(x, t)=T^{*}(x, t), \quad t \geq t^{*}
$$

provided that zero compatibility condition given in equation (2) are satisfied.

The control time $t^{*}$ is the minimum time for the propagation of the control law from the pump at the tube's inlet to the sensor located at the tube's outlet. It is given by

$$
t^{*}=\frac{1}{q_{\min }} .
$$

PROOF. The proof of proposition 3.1 is equivalent to the problem of finding the conditions of existence and uniqueness of equation (1)'s solution. This is obtained directly from the work of [20], Theorem 3.1 and its extinction in Proposition 5.1.

Note that in this reachability result, unlike Proposition 2.1, only the zero compatibility condition is needed. No further regularity conditions are required.

Remark 3.1 Proposition 3.1 states that to reach the target profile, this latter needs to be chosen such that it is at least continuous in time and of finite energy in space since the pump volumetric flow rate $q(t)$ can be piecewise constant in time (which corresponds for example to manipulations where the flow is maintained at a certain level for a period of time then switched to another). In practice, inter alia, the knowledge of the lower and upper bounds $q_{\min }, q_{\max }$ (respectively) should also be taken into consideration when choosing an admissible magnitude of the target profile. This is generally achieved at the reference governor stage (which is beyond the present work).

\section{Adaptive control design}

As presented in Subsection 2.2, the objective is to find the control input $q(t)$ under assumptions (i)-(iv) which stabilizes the average tracking error $\delta(t)$ defined in (6). The 
variable $\delta(t)$ represents the $L^{1}$-norm of the tracking error and thus it may be regarded as an energy-like variable.

The idea of choosing this energy-like concept $\delta(t)$ as the controlled variable was first presented in [16]. However, it was based on the assumption that distributed measurements of $T(x, t)$ are available. Moreover as it was presented, the convergence result was only established for $\delta(t)$ and not for the tracking error. In [15] the control strategy proposed in [16] was enhanced by developing conditions under which the stabilization of $\delta(t)$ ensures the stabilization of the tracking error. In addition, the control design was based on a boundary state observer. The global exponential stability of both the tracking error and the plant in closed-loop was proven using the Lyapunov approach. Note that both [16] and [15] assumed available measurements of the solar irradiance $f(t)$.

In this work we consider the practical case of unknown source term $f(t)$. We propose a new adaptive control method based on the boundary measurements of $T(x, t)$ and the design of an estimator of $f(t)$. This control method will guarantee the global asymptotic practical convergence of the tracking error.

Definition: The boundary and initial data generate persistent excitations to the PDE model (1) if the output $y(t)=T(1, t)$ is non-zero for a.e $t \geq 0$.

More details on the PE property for PDEs can be found in [24] and references therein. In [25], it was stated that, unlike ODEs, in PDEs even a constant input can generate a persistent excitation.

In [15], the observer-based energy-like control law was given by the following theorem.

Theorem 4.1. Let the state observer be given by

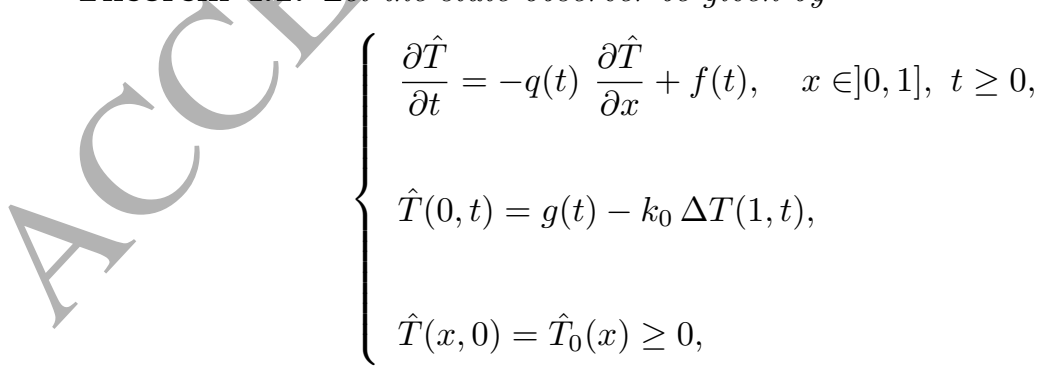

where $\hat{T}$ is the observer state estimate, $\Delta T(x, t)=\hat{T}(x, t)-T(x, t)$ is the state estimation error and $\Delta T(1, t)$ is the correction term (output error). 
Let the average tracking error be given by

$$
\hat{\delta}(t)=\int_{0}^{1}\left(T^{*}(x, t)-\hat{T}(x, t)\right) d x
$$

Consider the PDE given by (1) subject to assumptions (i)-(iv). Let $\hat{T}$ be given by PDE (16). The control input $q(t)$ given by

$$
q(t)=\frac{1}{(T(1, t)-T(0, t))}\left[f(t)-\int_{0}^{1} \frac{\partial T^{*}}{\partial t} d x-k_{p} \hat{\delta}(t)\right]
$$

ensures the exponential convergence of both the tracking error $\hat{\delta}(t)$ and the state estimation error $\Delta T(x, t)$ (in the $L_{2}-$ norm) for $t \geq t^{*}$ where $t^{*}$ is defined in $(12)$.

Proof. The proof of Theorem 4.1 was presented in [15].

\subsection{Analysis of the state and input estimator}

In this work, since the solar irradiance is unknown the state observer presented in (16) is modified, where the solar irradiance $f(t)$ is now estimated using an adaptation law. The new state-observer writes as

$$
\left\{\begin{array}{l}
\frac{\partial \hat{T}}{\partial t}=-q(t) \frac{\partial \hat{T}}{\partial x}+\hat{f}(t), \quad x \in[0,1], t \geq 0 \\
\hat{T}(0, t)=g(t)-k_{0} \Delta T(1, t), \\
\hat{T}(x, 0)=\hat{T}_{0}(x) \geq 0
\end{array}\right.
$$

where the state estimation error $\Delta T$ and the input estimation error $\Delta f$ are given by

$$
\left\{\begin{array}{l}
\Delta T(x, t)=\hat{T}(x, t)-T(x, t), \\
\Delta f(t)=\hat{f}(t)-f(t)
\end{array}\right.
$$

and where $\hat{f}$ is the estimated solar irradiance given by

$$
\left\{\begin{array}{l}
\frac{d \hat{f}}{d t}=-\sigma \hat{f}(t)-\vartheta\left(\frac{2}{\mu} \Delta T(1, t)-h(t)\right), \\
h(t)=-\frac{\mu_{f}^{2}}{\mu_{f}|\hat{f}(t)|+\epsilon_{f}} \hat{f}(t), \\
\hat{f}(0)=\hat{f}_{0} \geq 0, \quad \mu_{f} \geq\left|f_{e}(t)\right|, f_{e}(t)=-\frac{\sigma}{\vartheta}\left(f(t)+\frac{\dot{f}(t)}{\sigma}\right) .
\end{array}\right.
$$


where $\sigma>0$ introduces the so-called $\sigma$-modification term. The parameters $\mu$ and $k_{0}$ are chosen such as

$$
\mathrm{e}^{-\mu}-k_{0}^{2} \geq 0
$$

Theorem 4.2. Provided that $t \geq t^{*}$ ( $t^{*}$ defined in (12)), system (1) is persistently) excited and assumptions (i)-(iv) are satisfied, the state and input estimators given in equations (16)-(18)ensure the uniform ultimate boundedness of the states $L_{2}$-norm and input estimation errors. The uniform bound denoted $v$ can be made arbitrary small by choosing the adaptation gain $\vartheta$ sufficiently large.

Proof. From equations (1) and (16), the dynamics of the state estimation error $\Delta T$ are described by the following equation

$$
\left\{\begin{array}{l}
\frac{\partial \Delta T}{\partial t}=-q(t) \frac{\partial \Delta T}{\partial x}+\Delta f(t) \\
\Delta T(0, t)=-k_{0} \Delta T(1, t) \\
\Delta T(x, 0)=\hat{T}_{0}(x)-T_{0}(x)
\end{array}\right.
$$

Let us consider the following Lyapunov function

$$
V(t)=V_{1}(t)+V_{2}(t)
$$

where

$$
\left\{\begin{array}{l}
V_{1}(t)=\int_{0}^{1} \mathrm{e}^{-\mu x} \Delta T^{2}(x, t) d x, \\
V_{2}(t)=\frac{1}{2 \vartheta} \Delta f(t)^{2} .
\end{array}\right.
$$

The Lyapunov candidate function $V_{1}$ was first presented in [4] and [9], its derivative along the solution of systems (1)-(16) writes as

$$
\begin{aligned}
\dot{V}_{1}(t) & =2 \int_{0}^{1} \mathrm{e}^{-\mu x} \Delta T(x, t) \frac{\partial \Delta T}{\partial t} d x \\
& =-q(t) \int_{0}^{1} \mathrm{e}^{-\mu x} \frac{\partial \Delta T^{2}}{\partial x} d x+2 \Delta f(t) \int_{0}^{1} \mathrm{e}^{-\mu x} \Delta T(x, t) d x .
\end{aligned}
$$


Using the integration by part and the boundary condition in (16), we obtain

$$
\dot{V}_{1}(t)=-\mu q(t) V_{1}(t)-q(t)\left(\mathrm{e}^{-\mu}-k_{0}^{2}\right) \Delta T(1, t)^{2}+2 \Delta f(t) \int_{0}^{1} \mathrm{e}^{-\mu x} \Delta T(x, t) d x .
$$

Let us focus now on the third term of equation (24). Since $t \geq t^{*}$ then $x \leq \int_{0}^{t} q(\tau) d \tau$ and therefore the analytic solution of $\Delta T$ writes as

$$
\Delta T(x, t)=-k_{0} \Delta T(1, t-\gamma)+\int_{0}^{\gamma} \Delta f(\tau) d \tau,
$$

where $\gamma$ was already introduced in equation (9). This analytic solution in equation (25) is obtain using the method of charactiristics as in equation (8). From equation (25), since $\gamma$ is a positive increasing function then if we assume that $\Delta f$ is positive, the integral term $\int_{0}^{\gamma} \Delta f(\tau) d \tau$ will be increasing. Therefore, the term $\Delta T(x, t)+k_{0} \Delta T(1, t-\gamma)$ will be also positive and increasing. This implies that $\Delta T$ is increasing. Thus its space derivative is positive and the product $\Delta f(t) \frac{\partial \Delta T}{\partial x}$ is positive.

In the other hand, if we assume that $\Delta f$ is negative, the integral $\int_{0}^{\gamma} \Delta f(\tau) d \tau$ will be decreasing. Therefore, the term $\Delta T(x, t)+k_{0} \Delta T(1, t-\gamma)$ will be also decreasing. This implies that $\Delta T$ is decreasing and its space derivative is negative. The product $\Delta f(t) \frac{\partial \Delta T}{\partial x}$ is then positive. From this analysis we conclude that the term $\Delta f(t) \frac{\partial \Delta T}{\partial x}$ is always positive.

Now, by applying integration by parts on the third term of equation (24), we can state that

$$
\begin{aligned}
& \Delta f(t) \int_{0}^{1} \mathrm{e}^{-\mu / x} \Delta T(x, t) d x=\frac{1}{\mu} \Delta f(t)\left(\Delta T(0, t)-\mathrm{e}^{-\mu} \Delta T(1, t)\right) \\
& +\frac{1}{\mu} \Delta f(t) \int_{0}^{1} \mathrm{e}^{-\mu x} \frac{\partial \Delta T}{\partial x} d x \\
& \leq \frac{1}{\mu} \Delta f(t)\left(\Delta T(0, t)-\mathrm{e}^{-\mu} \Delta T(1, t)\right)+\frac{1}{\mu} \Delta f(t) \int_{0}^{1} \frac{\partial \Delta T}{\partial x} d x \\
& \leq \frac{1}{\mu} \Delta f(t)\left(1-\mathrm{e}^{-\mu}\right) \Delta T(1, t)
\end{aligned}
$$

where in the first inequality we have used the fact that the product $\Delta f(t) \frac{\partial \Delta T}{\partial x}$ is positive. From the last inequality in (26) we can guarantee that

$$
\Delta f(t) \int_{0}^{1} \mathrm{e}^{-\mu x} \Delta T(x, t) d x \leq \frac{1}{\mu} \Delta f(t) \Delta T(1, t) .
$$


Now combining the derivatives of $V_{1}$ and $V_{2}$ we obtain

$$
\dot{V}(t) \leq-\mu q_{m i n}\|\Delta T(., t)\|_{2}^{2}+\frac{2}{\mu} \Delta T(1, t) \Delta f(t)+\frac{1}{\vartheta} \Delta f(t)(\dot{\hat{f}}(t)-\dot{f}(t))
$$

By replacing $\dot{\hat{f}}$ by its expression given in equation (18), equation (28) becomes

$$
\dot{V}(t) \leq-\mu q_{m i n}\|\Delta T(., t)\|_{2}^{2}+\frac{1}{\vartheta} \Delta f(t)(-\sigma \hat{f}(t)+\vartheta h(t)-\dot{f}(t))
$$

By replacing $\Delta f$ by its expression given in equation (17), we obtain the following, inequality

$$
\begin{aligned}
\dot{V}(t) & \leq-\mu q_{\text {min }}\|\Delta T(., t)\|_{2}^{2}-\frac{\sigma}{\vartheta} \Delta f^{2}(t)+\frac{\sigma}{\vartheta} f^{2}(t)+\frac{1}{\vartheta} f(t) \dot{f}(t)+|\hat{f}(t)|\left|f_{e}(t)\right| \\
& +\hat{f}(t) h(t)+h(t)|f(t)|,
\end{aligned}
$$

By recalling that $\mu_{f} \geq\left|f_{e}\right|$ and replacing $h(t)$ by its formula given in equation (18), inequality (30) becomes

$$
\begin{aligned}
\dot{V}(t) & \leq-\mu q_{\min }\|\Delta T(., t)\|_{2}^{2}-\frac{\sigma}{\vartheta} \Delta f^{2}(t)+\frac{\sigma}{\vartheta} f^{2}(t)+\frac{1}{\vartheta} f(t) \dot{f}(t) \\
& +\mu_{f}|\hat{f}(t)|-\hat{f}(t) \frac{\mu_{f}^{2}}{\mu_{f}|\hat{f}(t)|+\epsilon_{f}}\left|\hat{f}(t)+\frac{\mu_{f}^{2}}{\mu_{f}|\hat{f}(t)|+\epsilon_{f}}\right| \hat{f}(t)|| f(t) \mid .
\end{aligned}
$$

which after simplification becomes

$$
\dot{V}(t) \leq-M V(t)+v(t)
$$

where $M=\min \left(q_{\min } \mu, \sigma\right)$ and $v(t)$ is a region defined by

$$
v(t)=\frac{\sigma}{\vartheta} f^{2}(t)+\frac{1}{\vartheta} f(t) \dot{f}(t)+\epsilon_{f}+\mu_{f}|f(t)|,
$$

From inequality (32), by considering the upper-bound $v_{\infty}$ of the region $v(t)$ and using Gronwall's inequality we end up with the following inequality

$$
\left\{\begin{array}{l}
V(t) \leq V(0) \mathrm{e}^{-M t}+v_{\infty} \\
v_{\infty}=\frac{\sigma}{\vartheta}\left|f^{2}(t)\right|_{\infty}+\frac{1}{\vartheta}|f(t) \dot{f}(t)|_{\infty}+\epsilon_{f}+\mu_{f}|f(t)|_{\infty}
\end{array}\right.
$$

and thus $\lim _{t \rightarrow \infty} V(t) \leq v_{\infty}$

Note that the region $v_{\infty}$ can be made arbitrary small by a suitable choice of the parameters $\sigma, \epsilon_{f}$ and $\vartheta$. However, this choice should take into consideration the state and 
parameter error convergence speed which depends on parameter $M$ (equation (31)). Note also that the knowledge of the bounds on the solar irradiance $f(t)$ and its derivative $\dot{f}(t)$ is not required. Furthermore, the standard assumption $\dot{f} \in L_{1}(0, t)$ (see e.g [17]) is not necessary. The term $h(t)$ accounts for the time variations of $f(t)$.

Proposition 4.3. For all initial state estimation errors $\Delta T(x, 0) \in L_{2}(\Omega),\|\Delta T(x, 0)\|_{2} \neq 0$, the minimum time convergence $\tau_{\text {min }}$ of state and input estimation errors to a region $\eta \geq v_{\infty}$ is given by

$$
\tau_{\text {min }}=\frac{1}{M} \ln \left(\left(\eta-v_{\infty}\right) / V(0)\right),
$$

PROOF. The proof is directly induced from inequality (34)

$$
V(0) \mathrm{e}^{-M t}+v_{\infty} \leq \eta \Rightarrow t \geq \frac{1}{M} \ln \left(\left(\eta-v_{\infty}\right) / V(0)\right)
$$

Proposition 4.3 provides a measure of the requested minimal time for the state and input estimation errors to converge to a region around $v(t)$ and which can be made arbitrary small by a careful choice of the parameters $\sigma, \epsilon_{f}$ and $\vartheta$.

Remark 4.1 In the case of a slowly time-varying input, using the same arguments and method presented in [26] and [27], the state estimator given by (16) and the input adaptation law given by (18) but without the term $h(t)$ ensure the asymptotic convergence of the state estimation error in the $L_{2}$-topology. By assuming that the system (1) is persistently excited, the asymptotic convergence of the input estimation error is also proved.

\subsection{Analysis of the adaptive control strategy}

Once the new state observer given by equation (16) and the input adaptation law given by equation (18) are defined and the convergence of the state and input estimation errors is analyzed, we can move to the study of the convergence of the system in closedloop, i.e using the control law defined by system (15) but with $\hat{q}(t)$ given by

$$
\hat{q}(t)=\frac{1}{(T(1, t)-T(0, t))}\left[\hat{f}(t)-\int_{0}^{1} \frac{\partial T^{*}}{\partial t} d x-k_{p} \hat{\delta}(t)\right]
$$

Compared to equation (15) the solar irradiance $f(t)$ is replaced by its estimate $\hat{f}(t)$. In this section, we will investigate the convergence of the average tracking error of the closed-loop system. 
Theorem 4.4. Consider the system (1) under assumptions (i)-(iv) with the controller (37), the state observer (16), the adaptation law (18), condition (19) and $t \geq t^{*}$. Then the solution $(T, \hat{f})$ is bounded for all $x \in[0,1], t \geq 0$. The $L_{2}$-norm of the state estimation error $\|\Delta T\|_{2}$, the source term error $\Delta f$ and the average tracking error $\delta(t)$ are globally practically asymptotically stable.

Proof. The proof is based on Lyapunov theory. Let us consider the following Lyapunov candidate function.

$$
\left\{\begin{array}{l}
V_{*}(t)=V(t)+V_{3}(t) \\
V_{3}(t)=\frac{1}{2} \delta^{2}(t)
\end{array}\right.
$$

where $V$ was defined as in (22). Taking into consideration the fact that

$$
\delta(t)=\hat{\delta}(t)+\int_{0}^{1} \Delta T(x, t) d x
$$

and the control law defined in (37), the derivative of $\delta$ is given by

$$
\dot{\delta}(t)=\Delta f(t)-k_{p} \hat{\delta}(t)
$$

Thus the derivative of $V_{3}$ is given by

$$
\begin{aligned}
\dot{V}_{3}(t) & =-k_{p} \delta^{2}(t)+k_{p} \delta(t) \int_{0}^{1} \Delta T(x, t) d x+\delta(t) \Delta f(t), \\
& \leq-\frac{\left(k_{p}-\vartheta\right)}{2} \delta^{2}(t)+\frac{k_{p}}{2}\|\Delta T(., t)\|_{2}^{2}+\frac{1}{2 \vartheta} \Delta f^{2}(t) .
\end{aligned}
$$

The last inequality was obtained using Young's inequality. Using equations (32), we know that

$$
\dot{V}(t) \leq-\mu q_{\min }\|\Delta T(., t)\|_{2}^{2}+v(t),
$$

and the following inequality of the derivative of $V_{*}$ can be induced

$\dot{V}_{*}(t) \leq-\frac{\left(k_{p}-\vartheta\right)}{2} \delta^{2}(t)-\left(\mu q_{m i n}-\frac{k_{p}}{2}\right)\|\Delta T(., t)\|_{2}^{2}-\frac{\epsilon}{2 \vartheta} \Delta f^{2}(t)+\frac{1+\epsilon}{2 \vartheta} \Delta f^{2}(t)+v(t)$.

If the parameters $k_{p}$ and $\mu$ are chosen such as

$$
k_{p}>\vartheta \text { and } \mu>\frac{k_{p}}{2 q_{\min }},
$$


then

$$
\begin{aligned}
\dot{V}_{*}(t) & \leq-\beta V_{*}(t)+\frac{1+\epsilon}{2 \vartheta} \Delta f^{2}(t)+v(t) \\
& \leq-\beta V_{*}(t)+\frac{3+\epsilon}{2} v(t) .
\end{aligned}
$$

where $\beta=\min \left(k_{p}, \mu q_{\min }, \epsilon / 2\right)$ and the last inequality was deduced from inequality (34). Using the same arguments as those employed in the proof of theorem 4.2, we have

$$
\left\{\begin{array}{l}
V_{*}(t) \leq V_{*}(0) \mathrm{e}^{-\beta t}+\Gamma_{\infty} \\
\Gamma_{\infty}=\frac{3+\epsilon}{2} v_{\infty}
\end{array}\right.
$$

and thus $\lim _{t \rightarrow \infty} V(t) \leq \Gamma_{\infty}$. The region $\Gamma_{\infty}$ can be made arbitrary small by a suitable choice of the parameters $\sigma, \epsilon_{f}$ and $\vartheta$. In conclusion, we have shown the global practical asymptotic stability of the tracking error $\delta(t)$, the input error $\Delta f$ and of the $L_{2}$-norm of the state error $\Delta T$.

From inequality (45), the input-to-state stability of the controller with respect to signals $f(t)$ and $\hat{f}(t)$ is directly inferred.

Remark 4.2 The choice of a high adaptation gain $\vartheta$ reduces the size of the region $v$ but in the same time may induce high-frequency oscillations in both the estimation process and the control signal (see conditions (43)).

Remark 4.3 Note that in the proof of Theorem 4.4 the term $\left(\frac{\sigma}{\vartheta} \Delta f(t)\right)$ was not included to keep free choice of the parameters $\sigma$ and $\vartheta$ and use them in the tuning of the region of convergence $v_{\infty}$. Also, we have applied the simple version of Young's inequality to avoid introducing extra parameters which would complicate the convergence analysis without modifying its final conclusion.

\section{Simulation results}

For simulation, we consider a one-loop parabolic solar collector trough. It consists of a parabolic mirror which allows to gather the solar irradiance and concentrate it on an absorber tube which is placed on its focal line. Through this tube a fluid flows and heat 
is transferred to it. A sun-tracking algorithm revolves the mirror to follow the sun along its daily cycle. The heated fluid is then either directed to a storage tank or transported to feed a heat exchanger to produce heat stream for a turbine or as the primary energy source in a solar desalination plant [28], [1].

To prove the usefulness of our adaptive controller design, we propose for simulation two examples. Example 1 deals with the case of a slowly time-varying source term (input) $f(t)$ whereas Example 2 considers a fast time-varying source term. For both cases, the following simulation conditions are adopted.

\subsection{Simulation conditions}

Motivated by practical applications of the solar collector in [28] and [29], we select an almost piece-wise constant in time target profile $T^{*}$ as illustrated in Figure 1 . Since the temperature variations cannot be stiff, we choose a smooth slope for $T^{*}$.

For each time interval $\Delta_{i}=\left[t_{i}, t_{i+1}\right], i=\{1,2,3\}$, the profile $T_{i}^{*}(x, t)$ is generated using the steady-state solution of equation (1) as follows

$$
\begin{aligned}
& T_{i}^{*}(x, t)= \begin{cases}\left(T^{*}(0)+x\left(T_{i}^{*}(1)-T^{*}(0)\right)\right) \mathbb{1}(t), \quad t \in \Delta_{i}, \\
0 & \text { else }\end{cases} \\
& T^{*}(x, t)=\sum_{i=1}^{3} T_{i}^{*}(x, t),
\end{aligned}
$$

where $T^{*}(0)=20^{\circ}$ which corresponds to the case where the plant (solar collector) is fed by a storage tank at the ambient temperature. The target outlet temperature $T^{*}(1, t)$ is shown in Figure 1 (left panel). For practical issues, the inlet-outlet temperature gradient should not exceed $80^{\circ}$ [31].

Since $T^{*}(x, t)$ is the sum of $T_{i}^{*}(x)$ and $T^{*}(x, t=0)=T^{*}(x=0)=T(x, t=0)$ then $T^{*}(x, t)$ satisfies Proposition 2.2. Moreover, as it is depicted in this figure, the target temperature $T^{*}(x, t)$ belongs to our set of reachable states $C^{0}\left(0, t_{f} ; L^{2}(\Omega)\right)$ and thus exact reachability with respect to this set is feasible. The magnitudes of these parameters are coherent with what exists in practice, e.g. experimental data provided by AQUASOL desalination plant in [29].

The set of tuning parameters is chosen as follows $k_{p}=20, \sigma=0.1, k_{0}=10^{-3}, \mu=5$ and $\vartheta=10$ and the FEM (finite element method ) using quadratic B-splines functions 
is adopted for the implementation of the system in closed-loop in Simulink ${ }^{\circledR}$. Note that the tuning parameters where chosen by trial and error. Even if the PDE in equation (1) is linear, the estimator given by equations (16)-(18) is nonlinear and the control problem itself is bilinear. In this case it is very difficult to analyze analytically the effect of the tuning parameters. However, as stated by [30], p 367, in practice we noticed that increasing the tuning parameters will lead to faster parameter and control error convergence. But beyond a certain point, increasing the tuning gains will induce oscillations and slow down the convergence.
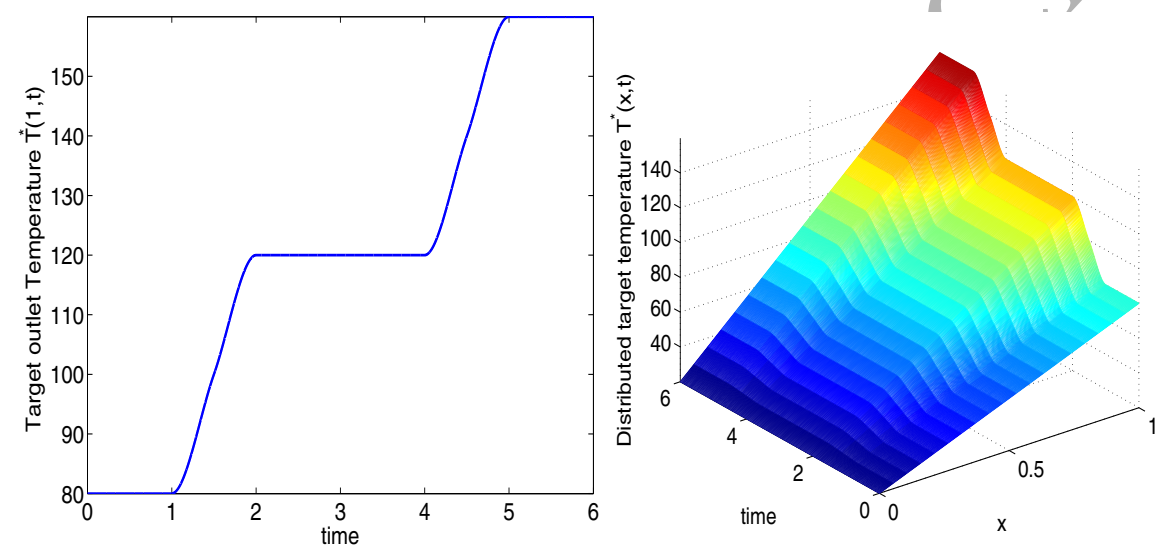

Figure 1: Outlet and distributed target temperature profiles

\subsection{Example 1: Case of a slowly time-varying $f(t)$}

We suppose that the one-loop parabolic solar collector trough is exposed to the solar irradiance shown in Figure 2 and the objective is to track the outlet temperature given in Figure 1. The distributed target temperature profile $T^{*}(x, t)$ is given in Figure 1 (right panel). The state observer in equation (16) is initialized such as the output $y(t)$ is nonzero and the PE condition is thus satisfied.

Using the control strategy proposed in (37), as it is illustrated in Figure 3, the profile of the controlled outlet temperature $T(1, t)$ follows the target outlet trajectory $T^{*}(1, t)$ with a tracking error less than $1 \%$ except at the ramp portions where it reaches the level of $2.7 \%$. Actually, the proposed control strategy in Figure 3 does not only guarantee the convergence of the outlet tracking error but also the convergence of the distributed one 


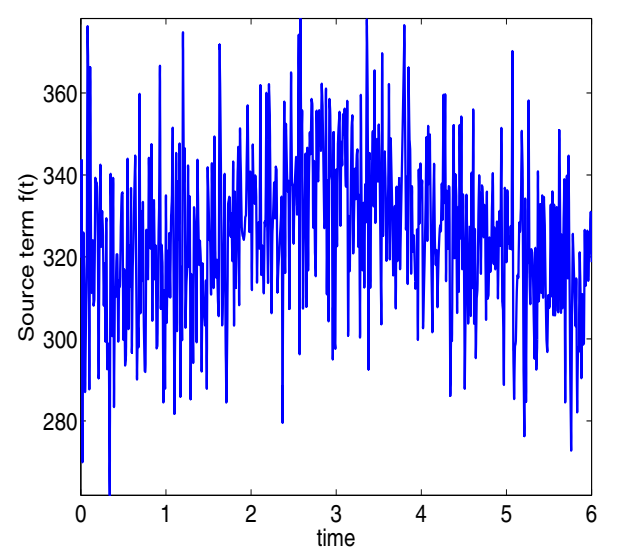

Figure 2: Source term $f(t)$

as it is shown in Figure 4. This figure represents the time evolution of the distributed tracking relative error for each spatial node in the FEM scheme.
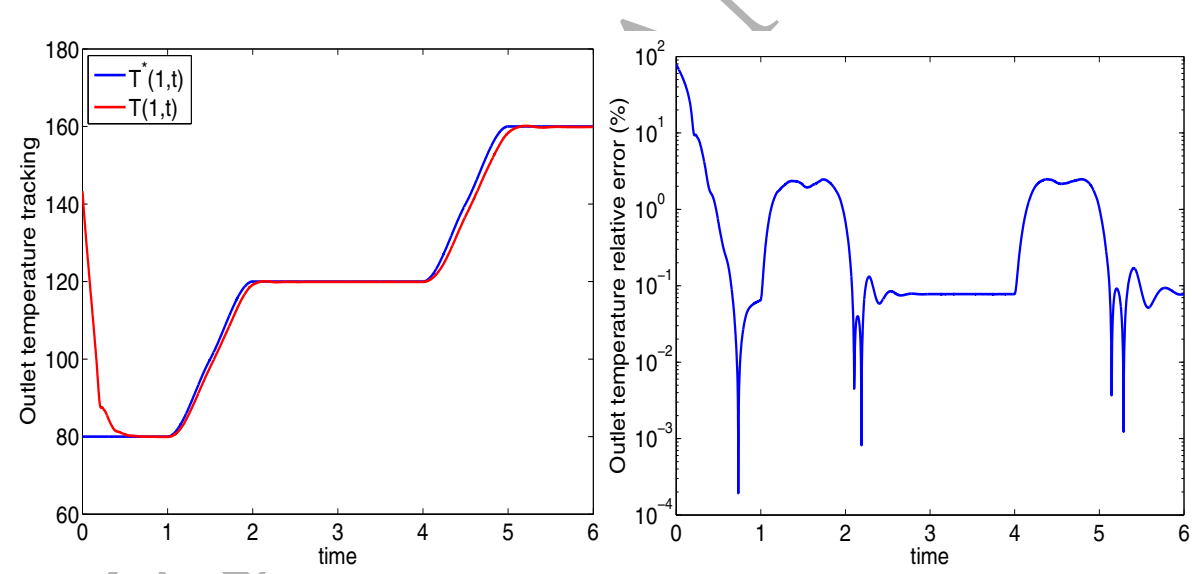

Figure 3: Outlet temperature tracking and its relative error.

In Figure 5 the control input $q(t)$ is presented together with the average tracking error $\hat{\delta}(t)$. We see from this figure that the control law is indeed in $C^{0}\left(0, t_{f}\right)$ and satisfies assumption (i). The average tracking error $\hat{\delta}(t)$ converges in finite time and is below 0.01 .

The source estimation error $\Delta f(t)$ converges in finite time and is less then $0.1 \%$ as it is 


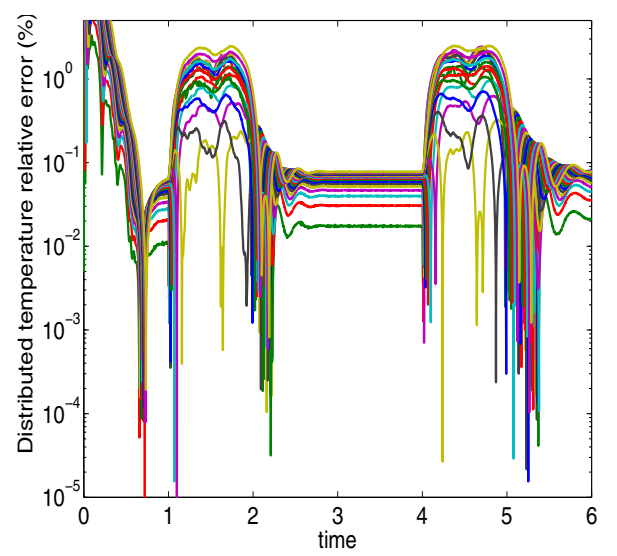

Figure 4: Distributed tracking relative error.
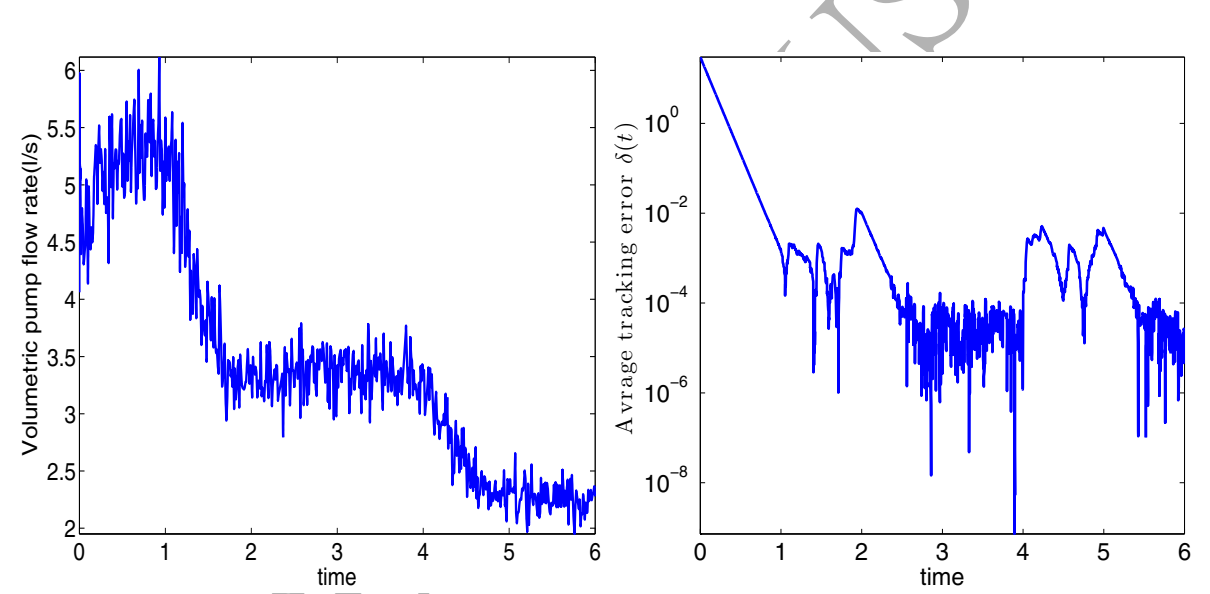

Figure 5: Control input $q(t)$ and the average tracking error $\delta(t)$

shown in Figure 6.

\subsection{Example 2: Case of a fast varying $f(t)$}

This case is proposed to test the efficiency of the proposed algorithm. We assume that the plant is exposed to the source term $f(t)$ shown in Figure 7 . The output $y(t)$ will be nonzero and the PE condition is thus satisfied. Note that such fast variations of

the solar irradiance are not frequent, yet they may appear for certain periods of time as it is shown for example in [29] and [31] (and references therein). The objective is still to track the target profile given in Figure 1. The estimator given by equation (16)-(18) 

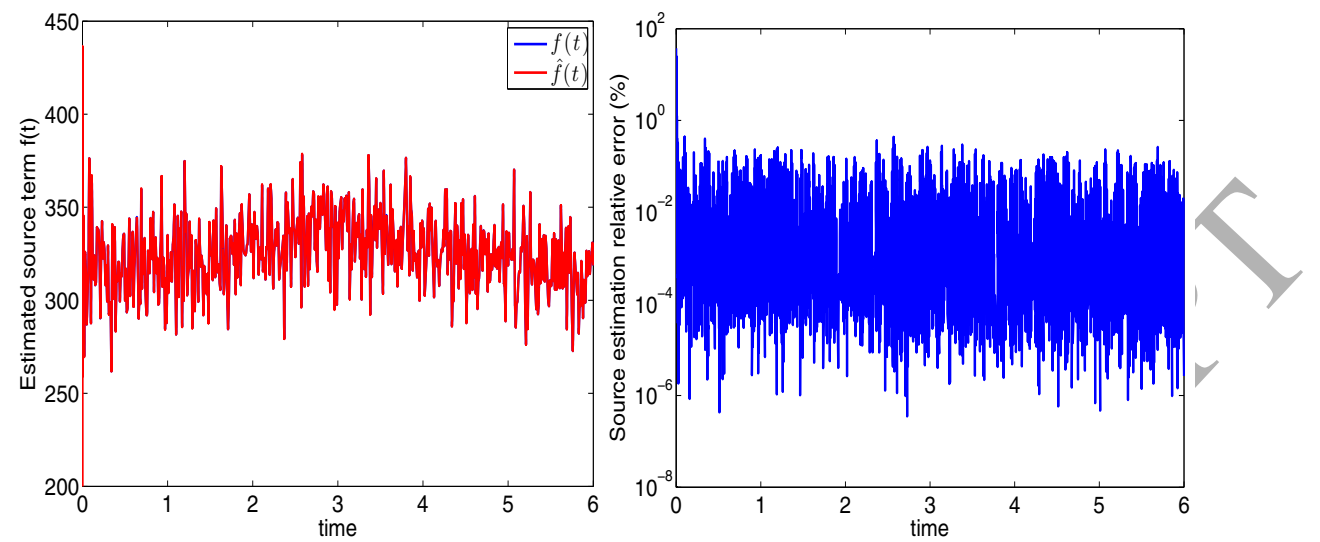

Figure 6: Estimated source term $f(t)$ and its relative estimation error (\%).

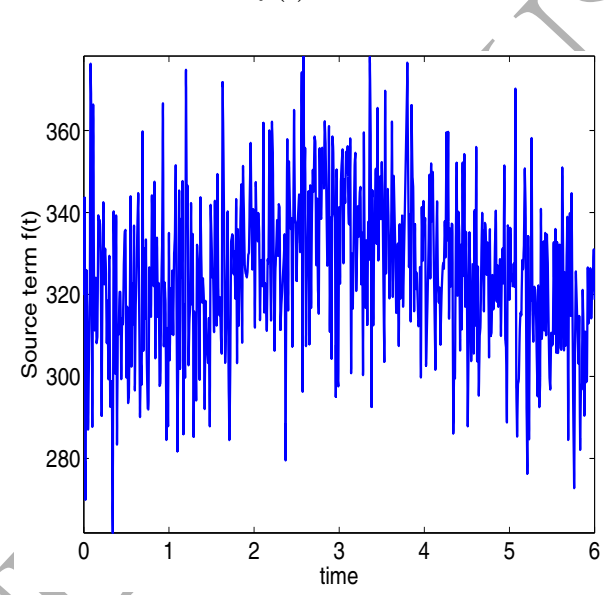

Figure 7: Source term $f(t)$.

together with the controller in equation (37) are implemented. The tracking error and the outlet tracking temperature are illustrated in Figure 8 where despite the unknown input fast variations $f(t)$ the controller is able to track the target profile with an error less than $1 \%$ in average. Also as it is shown in Figure 9, the distributed tracking error is converging rapidly and in finite time. It is in average less than $1 \%$. Again, each line represents the time evolution of the distributed tracking error for each spatial node in the FEM scheme.

Figure10 shows the volumetric pump flow rate $q(t)$. We see that it is directly affected 

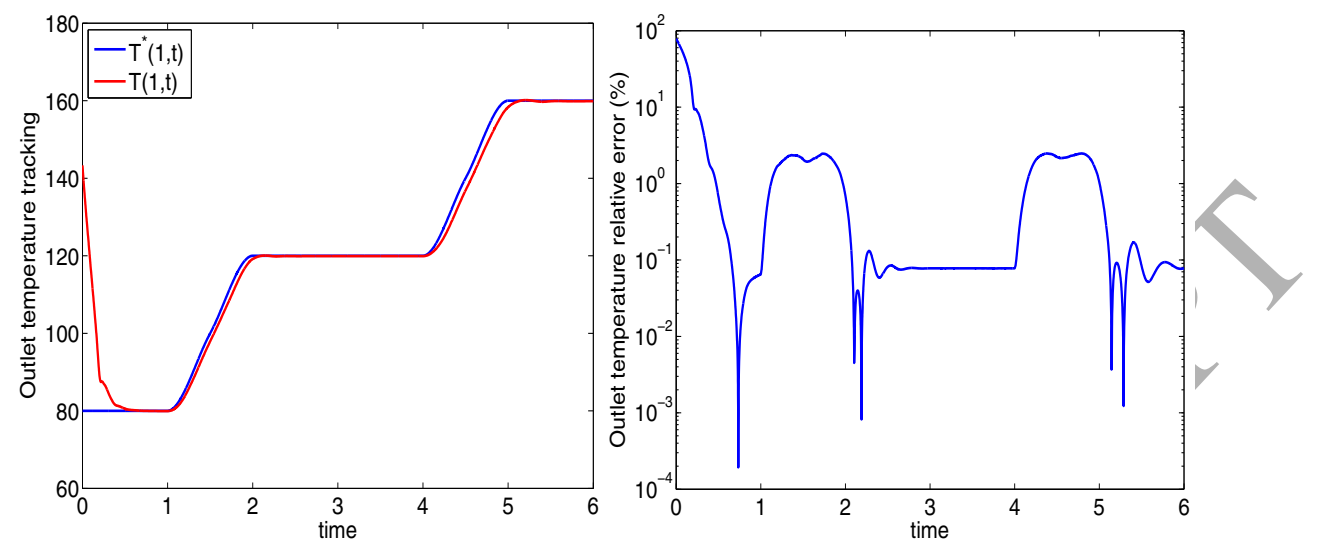

Figure 8: Outlet temperature tracking and its relative error.

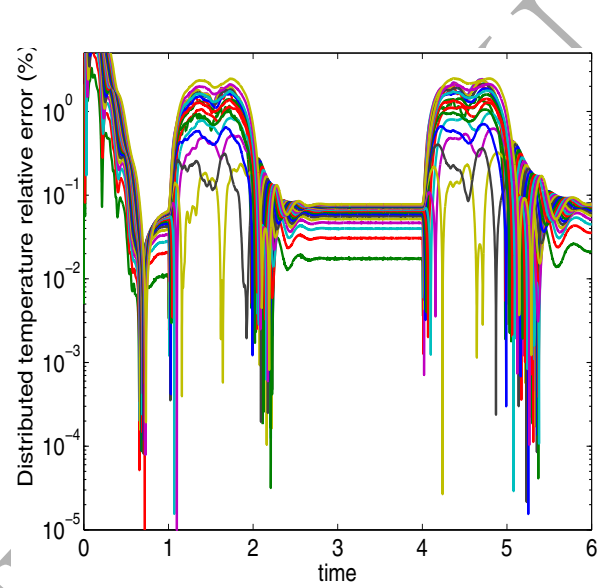

Figure 9: Distributed tracking relative error.

by the estimated profile of $f(t)$. Since the source term is fast varying, $q(t)$ needs also to vary rapidly in order to reach the control objective despite the fast variations of the unknown input $f(t)$. However, implementing such a control strategy in practice may not be feasible. This is why a post filtering is required in practice. The average tracking error $\hat{\delta}(t)$ (right panel) converges in finite time and is below 0.01 .

The convergence of the source estimation error $\Delta f(t)$ is shown in Figure 11 where we see that despite the fast variations of the source term $f(t)$ the algorithm is able to estimate it accurately. 

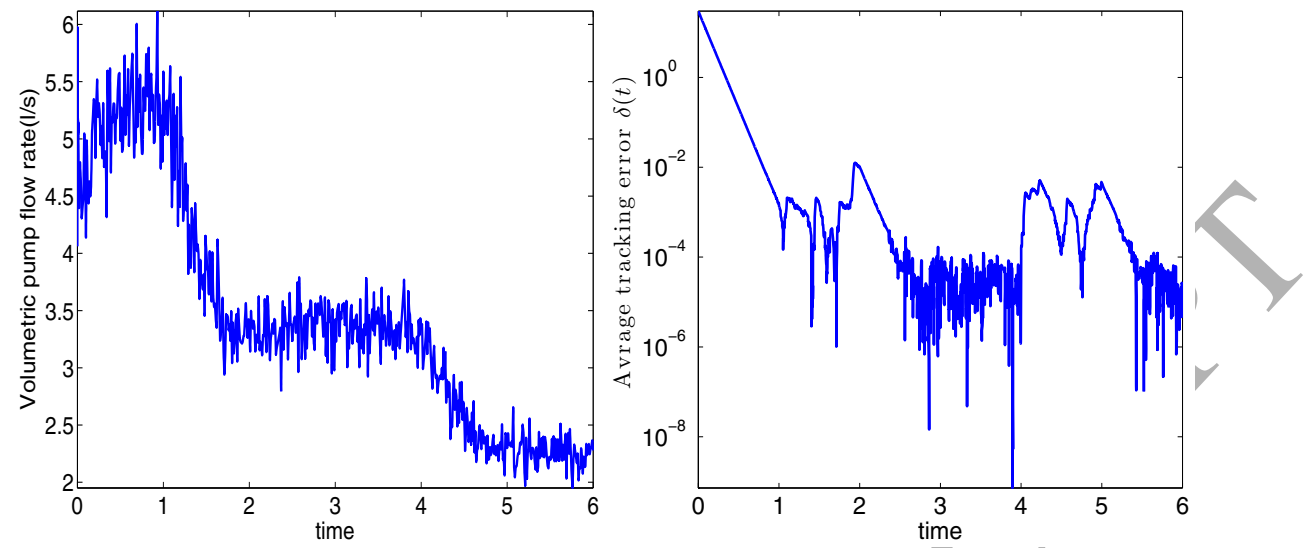

Figure 10: Control input $q(t)$ and the average tracking error $\delta(t)$

\section{Conclusion}

In this paper we addressed the problem of boundary tracking of a 1-D first-order hyperbolic PDE using bilinear control. As it was pointed and demonstrated in [23], exact controllability of bilinear distributed systems is not achievable this is why we started by defining the set of reachable states and then we formulated the control problem. It consists in designing the control law which allows the tracking of the outlet target temperature while assuming that the boundary state is known and the only available measurements are those obtained at the second boundary. The source term which is the input was assumed unknown and time-varying. By means of adaptive control methods, we were able to provide an adaptive control law which guarantees the global practical asymptotic stability under some conditions.

A potential application of this problem is the solar collector parabolic trough, where the flow velocity plays the role of the control input and the solar irradiance is the unknown source term.

Simulation results attest of the potential of the proposed control strategy. The estimation of the source term is not only necessary from a control perspective but even from a supervision point of view where the estimated source term can be used in selecting the target state at the reference governor stage.

In future works, first we will consider the robustness of the proposed control design 

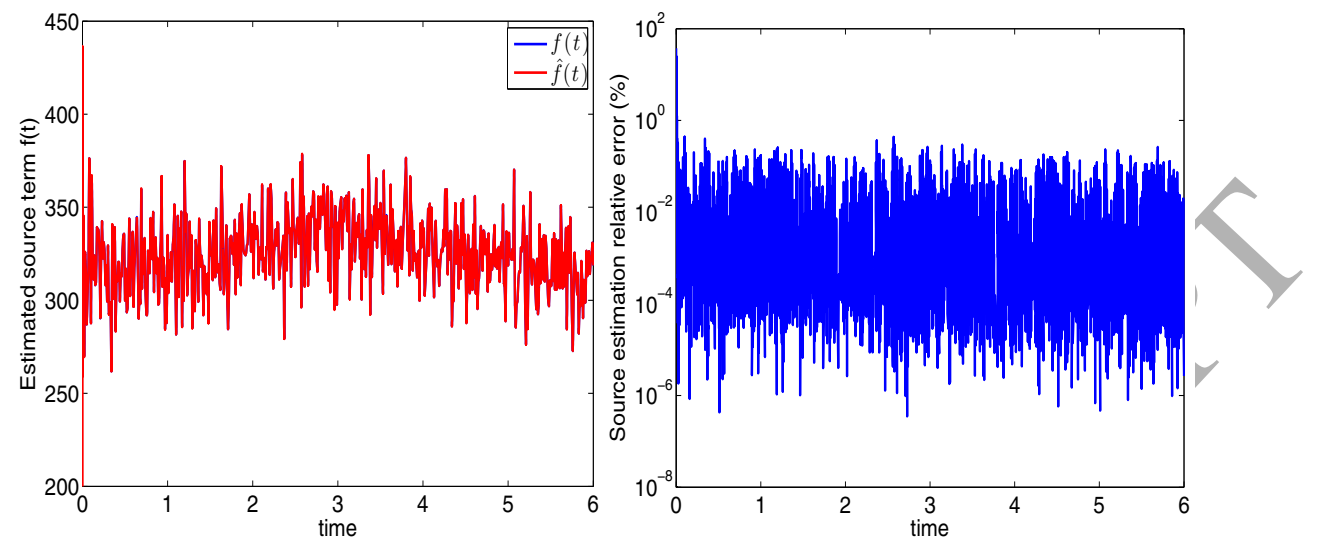

Figure 11: Estimated source term $f(t)$ and its relative estimation error (\%).

with respect to noisy measurements and then extended the problem to the case of a space and time varying source terms while taking into consideration the inlet temperature as an external disturbance.

\section{Acknowledgement}

Research reported in this publication has been supported by the King Abdullah University of Science and Technology (KAUST). The authors are very thankful to the anonymous reviewers and to the Associate Editor for their valuable comments which helped improving the presentation.

\section{References}

[1] E. F. Camacho, M. Berenguel, F. Rubio, D. Martinez, Control of solar enegy systems, SpringerVerlag London Limited, 2012.

2] P. D. Christofides, Nonlinear and robust control of PDE systems: Methods and application to transport-reaction processes, Springer Science+Business Media, LLC, 2001.

[3] E. Zuazua, Propagation, observation, and control of waves approximated by finite difference methods, SIAM Rev 47 (2005) 197-243.

[4] O. M. Aamo, J. Salvesen, B. A. Foss, Observer design using boundary injections for pipeline monitoring and leak detection, International Symposium on Advanced Control of Chemical Processes (2006) 53-58. 
[5] M. Krstic, A. Smyshlyaev, Backstepping boundary control for first-order hyperbolic pdes and application to systems with actuator and sensor delays, Systems Control Letters 57 (2008) 73-93.

[6] F. D. Meglio, R. Vazquez, M. Krstic, Stabilization of a system of $n+1$ coupled first-order hyperbolic linear pdes with a single boundary input, IEEE Transactions On Automatic Control 58 (2013) 3097-3111.

[7] P. D. Christofides, P. Daoutidis, Feedback control of hyperbolic PDE systems, AIChE J 42 (1996) 3063-3086.

[8] P. D. Christofides, P. Daoutidis, Robust control of hyperbolic PDE systems, Chemicál Engineering Science 53 (1998) 85-105.

[9] J. Coron, B. d'Andrea Novel, G. Bastin, A sitrict Lyapunov function for boundary control of hyperbolic systems of conservation laws., IEEE Transactions On Automatic Control 52 (2007).

[10] F. D. Meglio, D. Bresch-Pietri, U. J. F. Aarsnes, An adaptive observer for hyperbolic systems with application to under balanced drilling, Proceedings of the 19th IFAC World Congress (2014) 11391-11397.

[11] P. Bernard, M. Krstic, Adaptive output-feedback stabilization of non-local hyperbolic pdes, Automatica 50 (2014) 2692-2699.

[12] H. Wu, J. Wang, H. Li, Design of distributed h fuzzy controllers with constraint for nonlinear hyperbolic pde systems, Automatica 48 (2012) 2535-2543.

[13] J.-W. Wang, H.-N. Wu, H.-X. Li, Distributed fuzzy control design of nonlinear hyperbolic pde systems with application to nonisothermal plug-flow reactor, IEEE Transactions on Fuzzy Systems 19 (2011) 514-526.

[14] K. J. Yang, K. S. Hong, W. S. Yoo, O. K. Lim, Robust adaptive control of a time-varying heat equation with unknown bounded disturbances, JSME International Journal 44 (2001) 708-716.

[15] S. Mechhoud, T. M. Laleg-Kirati, Observer-based bilinear control of first-order hyperbolic pdes: Application to the solar collector, in: IEEE Control Conference on Decision and Control, 2015.

[16] T. A. Johansen, C. Storaa, Energy-based control of a distributed solar collector field, Automatica 38 (2002) 1191-1199.

[17] R. Marino, P. Tomei, Robust adaptive regulation of linear time-varying systems, IEEE Trans. on Auto. Control 45 (2000) 1301-1311.

[18] A. Pazy, Semigroups of linear operators and applications to partial differential equations, volume 44, Springer-Verlag New York, 1983.

[19] R. F. Stengel, Optimal Control and Estimation, Dover Publications, Inc., 1994.

[20] J. Rauch, F. J. Massey III, Differentiability of solutions to hyperbolic initial boundary value problems, Transactions of the AMS. 189 (1974) 303-318.

[21] C. Z. Xu, Exact observability and exponential stability of infinite-dimensional bilinear systems, Math. Control Signals Syst. 9 (1996) 73-93.

[22] M. Renardy, R. C. Rogers, An introduction to partial differential equations, Springer-Verlag New York, 2004.

[23] J. Ball, J. Marsden, M. Slemrod, Controllability for distributed bilinear systems, SIAM J. Control 
Optim. 20 (1982) 575-597.

[24] Y. Orlov, J. Bentsman, Adaptive distributed parameter systems identification with enforceable identifiability conditions and reduced-order spatial differentiation, IEEE Transaction On Automatic Control 45 (2000) 203-216.

[25] K. S. Hong, J. Bentsman, Direct adaptive control of parabolic systems: Algorithm synthesis and convergence and stability analysis, IEEE Transactions on Automatic Control 39 (1994) 2018-2033.

[26] M. A. Demetriou, Model reference adaptive control of slowly time-varying parabolic distributed parameter systems, in: Proceedings of the 33rd Conference on Decision and Control, Lake Buena Vista, FL, 1994, pp. 775-780.

[27] H. Banks, M. A. Demetriou, Adaptive parameter estimation of hyperbolic distributed parameter systems: Non-symmetric damping and slowly time varying systems, ESAIM: Control, Optimization and Calculus of Variations 3 (1998) 133-162.

[28] C. M. Cirre, J. C. Moreno, M. Berenguel, J. L. Guzman, Robust control of solar plants with distributed collectors, in: Proceedings of the 9h International Symposium on Dynamics and Control of Process Systems, Leuven, Belgium, 2010.

[29] C. O. Ayala, L. Roca, J. L. Guzman, J. E. Normey-Rico, M. Berenguel, L. Yebra, Local model predictive controller in a solar desalination plant collector field, Renewable Energy 36 (2011) 30013012.

[30] J. Slotine, W. Li, Applied nonlinear control, Prentice-Hall, Inc., 1991.

[31] G. Andrade, D. Pagano, J. Alvarez, M. Berenguel, A practical nmpc with robustness of stability applied to distributed solar power plants, Solar Energy 92 (2013) 106-122. 